\title{
Research Paper: \\ Comparison of Feeding Behaviors in Term and Preterm Infants at Six Months Corrected Age
}

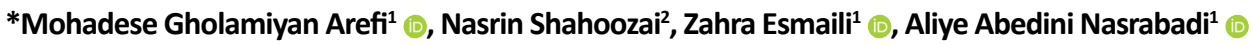

1. Department of Speech Therapy, Faculty of Rehabilitation Sciences, Zahedan University of Medical Sciences, Zahedan, Iran.

2. Department of Speech Therapy, Faculty of Rehabilitation Sciences, Mashhad University of Medical Sciences, Mashhad, Iran.

\begin{tabular}{|c|c|}
\hline $\begin{array}{l}\text { Use your device to scan } \\
\text { and read the article online }\end{array}$ & \\
\hline nrthe; & Citation Gholamiyan Arefi M, Shahoozai N, Esmaili Z, Abedini Nasrabadi A. Comparison of Feeding Behaviors in Term and Preterm \\
\hline 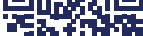 & Infants at Six Months Corrected Age. Jundishapur Journal of Medical Sciences. 2021; 20(2):140-149. https://doi.org/10.32598/ \\
\hline 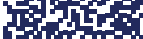 & JSMJ.20.2.5 \\
\hline astyct & dol'https://doi.org/10.32598/JSMJ.20.2.5 \\
\hline
\end{tabular}

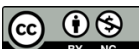

Received: 16 Feb 2020 Accepted: 20 Sep 2020 Available Online: 01 Jun 2021

Keywords:

Preterm infants, feeding behaviors, feeding problems

\section{A B STRACT}

Background and Objectives: This study compares feeding behaviors and problems of in term Infants and preterm infants at 6 months corrected age.

Subjects and Methods The study was of observational-sectional type (descriptive and analytic) and sampling was one-step cluster probability of the available population in the form of the whole number. Feeding behaviors of 90 infants, including 60 term infants and 30 preterm infants, were assessed by speech and language pathologists through interviews with parents and recorded in a questionnaire. The information in this questionnaire includes feeding behaviors, feeding problems, feeding period, infant weight at 2 , 4, 6 months and the age of the child to start complementary feeding, SPSS software version 24 was used to analyze the data. In all the present study, P-value less than $5 \%$ was considered as a significant level. Results The results showed that the two groups were significantly different in the mean score of feeding behavior $(P=0.001)$, feeding problems $(P=0.047)$ and weight gain $(P<0.001)$. While in feeding duration $(P=0.53)$ and Frequency of feeding $(P=0.1)$ there was no significant difference. Of the 90 infants studied, more than $80 \%$ of them started feeding in the form of puree in the age group of 4-6 months.

Conclusion Premature infants have more feeding problems and less weight gain than normal infants. It is recommended that the speech and language pathologist evaluate and intervene appropriately for oral skills and nutrition of premature infants admitted to the neonatal intensive care unit.

\section{* Corresponding Author:}

Address: Department of Speech Therapy, Faculty of Rehabilitation Sciences, Zahedan University of Medical Sciences, Zahedan, Iran.

Tel: +98 (54) 33295835

E-Mail: gholamiyan.m@gmail.com 


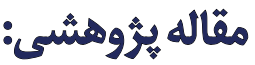 \\ مقايسه رقتار هاى تغذيهاى شيرخواران نارس 7 ماهله اصلاحشده وشيرخوار ان طبيعى شهر زاهدان}

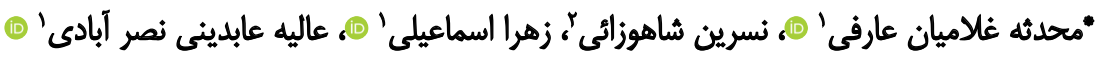

1. كروه تفتاردرمانى، دانشكده توانبخشى، دانشكاه علوم يرٔشكى زاهدان، زاهدان، ايران.

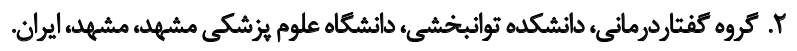

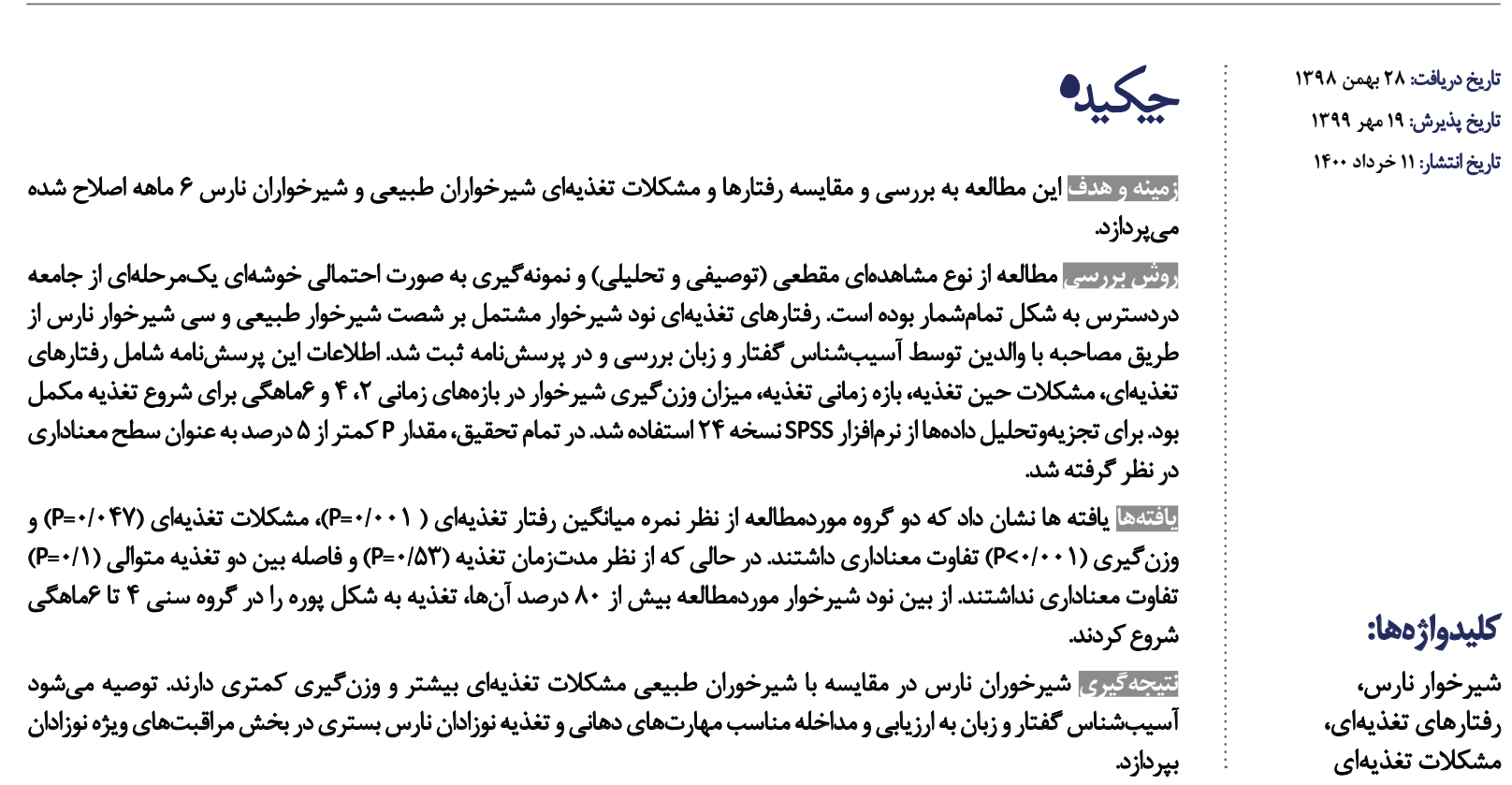

است. با اين حال تولد زودرس هنوز هم يكى از دلايل عمده

مقدمه

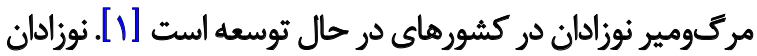

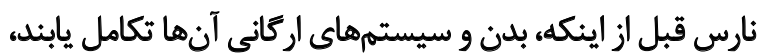

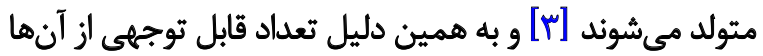

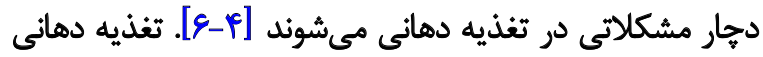

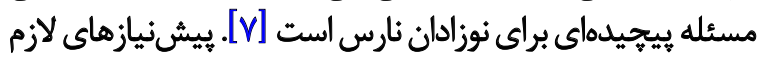

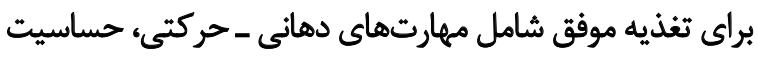

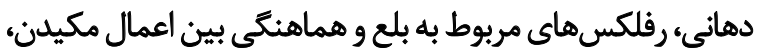

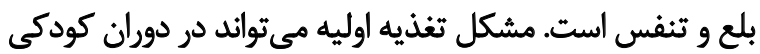

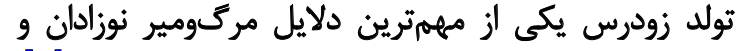

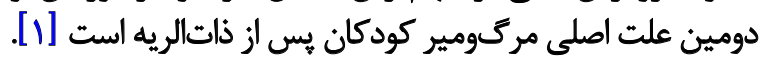

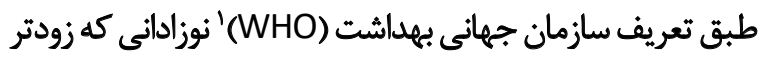

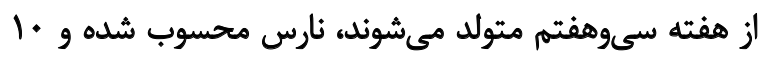

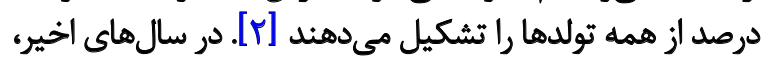

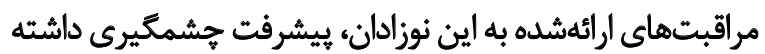

1. World Health Organization

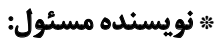

محدثه غلاميان عارفى في مئده

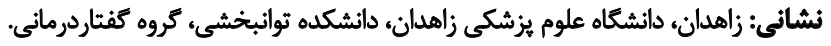
تلفي: واياثامه: gholamiyan.m@gmail.com 
آنها انجام دادند، مشخص شد كه اقليت اين شيرخواران در

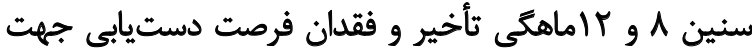

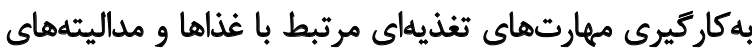

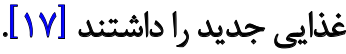

در مطالعه كه توسط اميرشاهى و همكاران با هدف بروسى

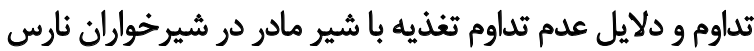

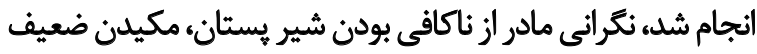

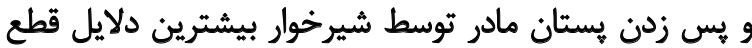

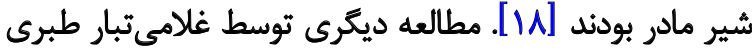

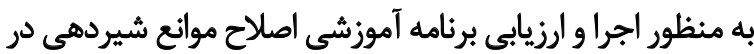

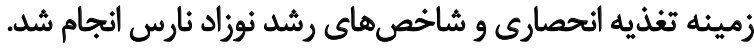

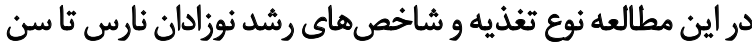

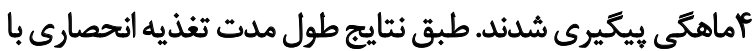

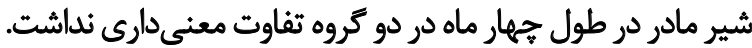

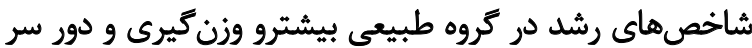

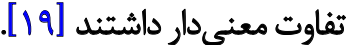

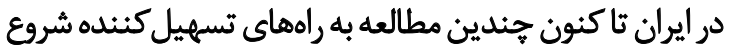

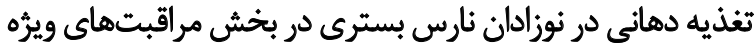

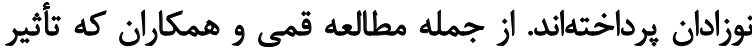

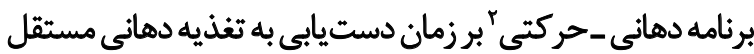

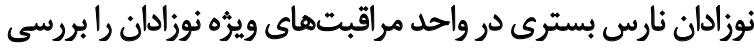

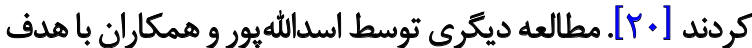

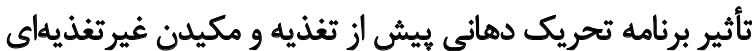

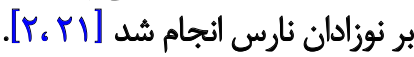

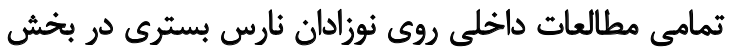

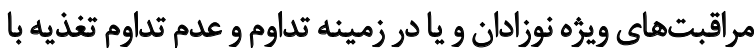

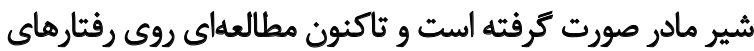
تغذيهاى شيرخواران نارس و تفاوت آنها

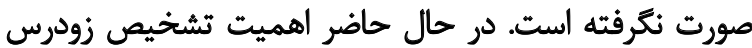

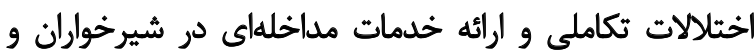

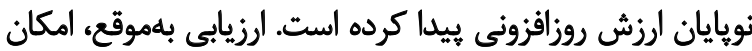

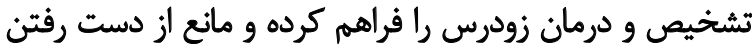

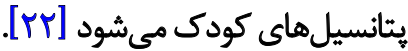

با توجه به اينكه طبق تعريف انجمن شنوايي و كفتار

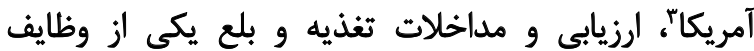

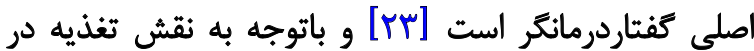

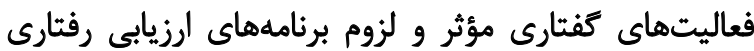

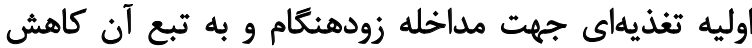

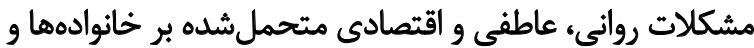
نيز با توجه به نبود مطالعهى در اين زمينه در ايران، محققان بران

2. Oral Motor Intervention (PIOMI) 3. ASHA

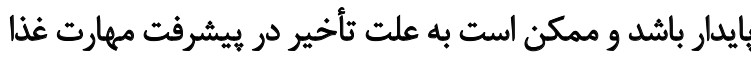

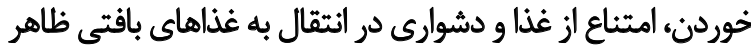

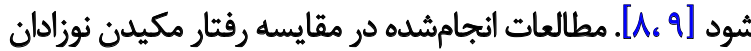

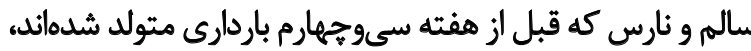

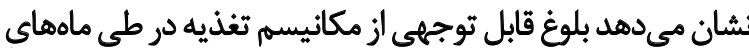

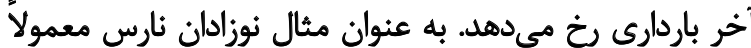

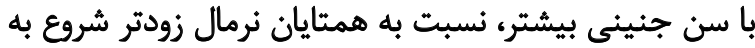

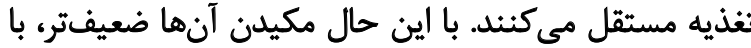

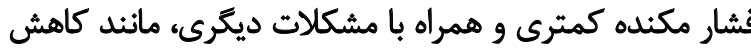

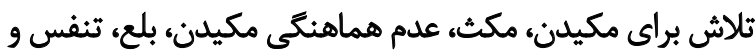

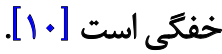
نوزادان نارس در مقايسه با نوزادان طبيعى يس از تولد به بهانه

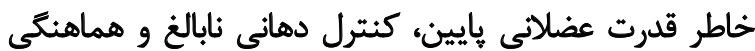

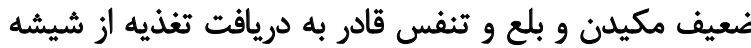

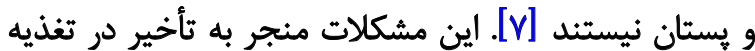

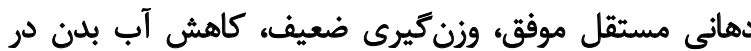

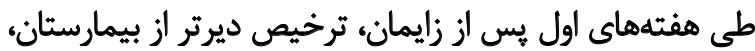

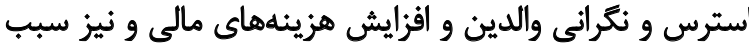

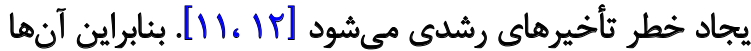

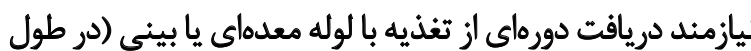

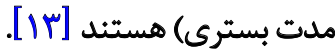

در مطالعهاى كه توسط بروسكو و همكاران باهدف بررسى رشد

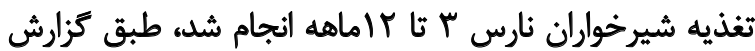

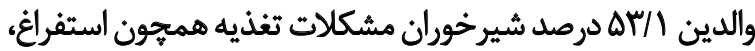

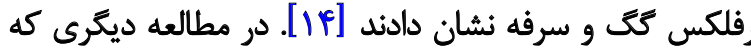

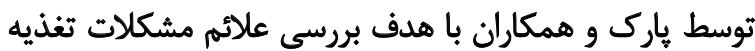

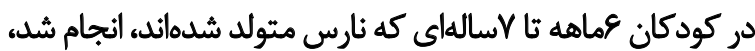

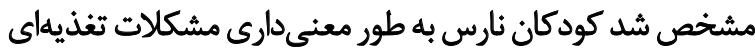

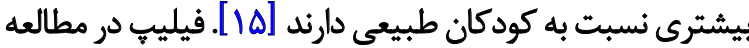

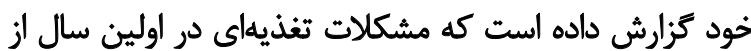

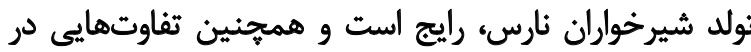

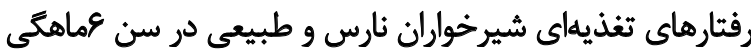

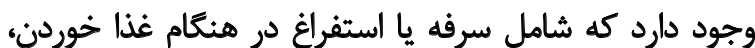

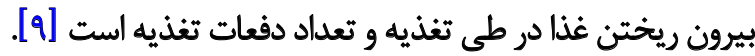
ياّكليارو و همكاران در مطالعه خود نشان دادئد نوزادان نارسى

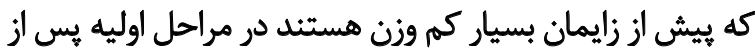

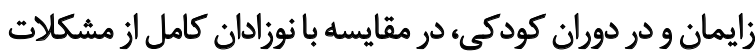

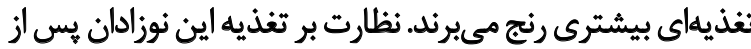

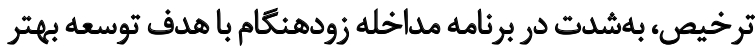

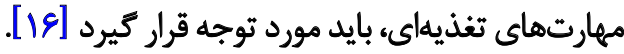
طى مطالعالى كه بريدهام و همكارانش جهت سنجش كارايى مهارت تغذيهاى شيرخواران نارس در طول ماركان اولين سال زندكى 
به دليل محدوديت سني موردنظر در مطالعه (وماهه اصلاحشدهاه

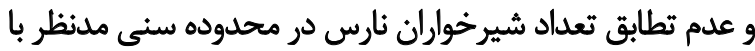

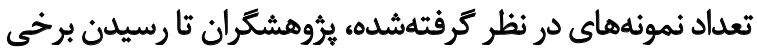

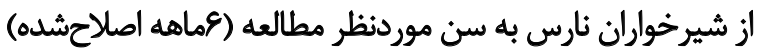

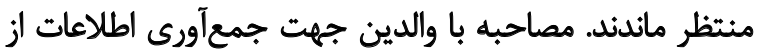

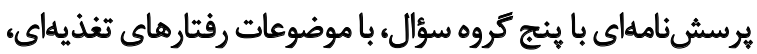

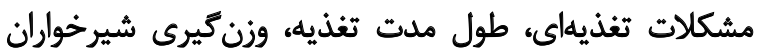

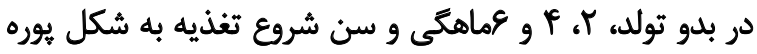
استفاده شد.

درنهايت يس از تردآورى دادههاى خام، اطلاعات وارد نرمافزار نسخه SPSS

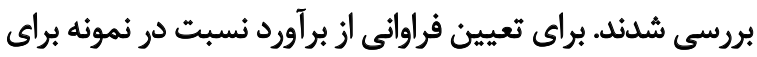

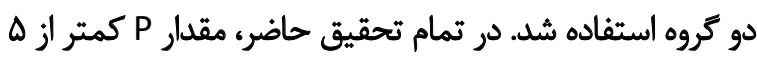

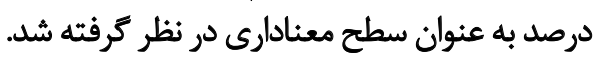

bevill

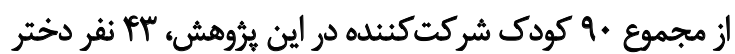

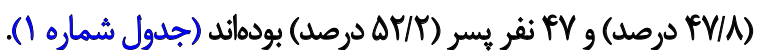
نتيجه آزمون كايدو نشان داد كه نسبت جنس در دارئ داري

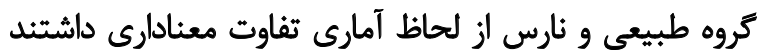
(P=×/FV)

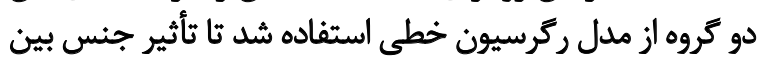

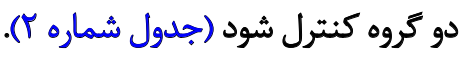

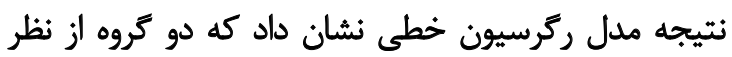

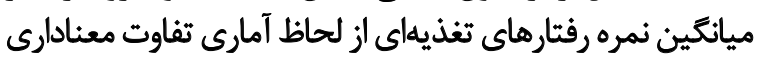

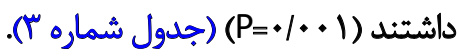

مدل ركرسيون خطى نشان داد كه با كنترل اثر جنس، ميانكين

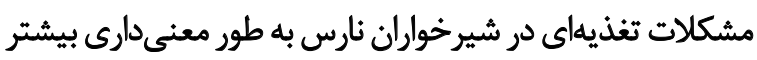

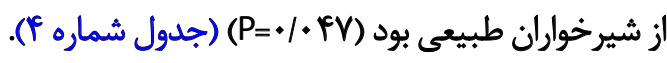

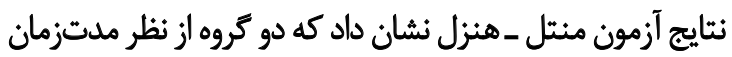

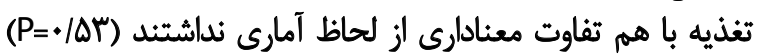

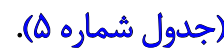

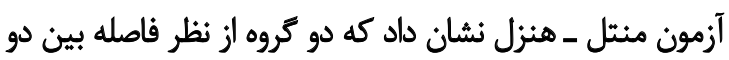

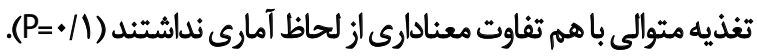

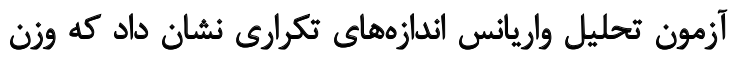

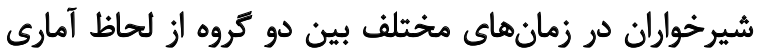

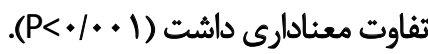

As

هدف اين مطالعه مقايسه رفتارهاى تغذيهاى شيرخواران
بر آن شدند ثا رفتارهاى تغذيهاى شيرخواران طبيعى و نارس

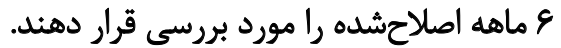

\section{ورش بروسي}

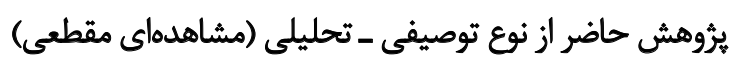

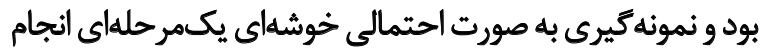

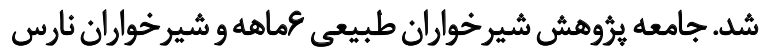

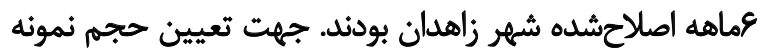

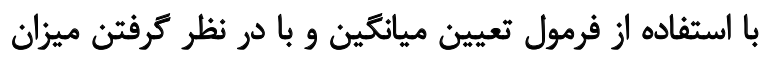

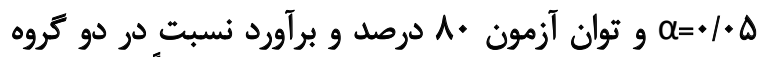

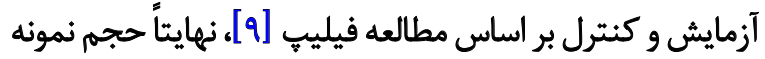

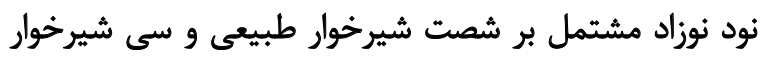

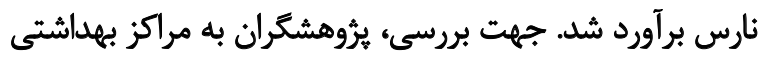

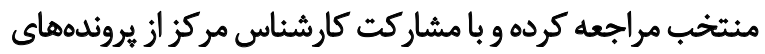

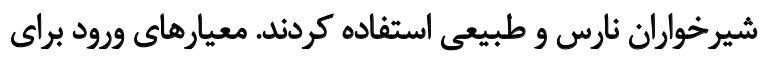

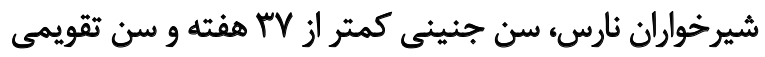

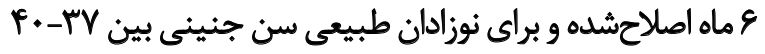

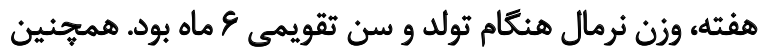

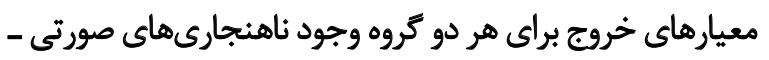

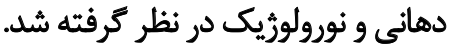

به دليل حضور شيرخواران نارس در مطالعه و نبود اطلاعات

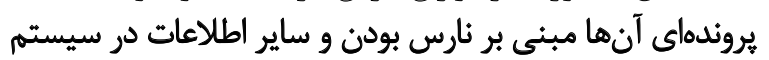

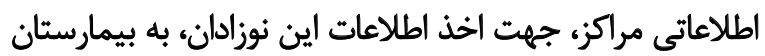

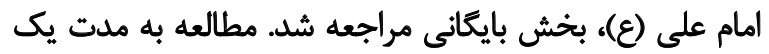

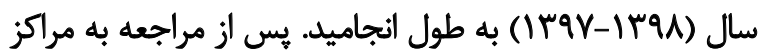

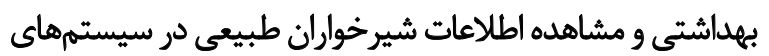

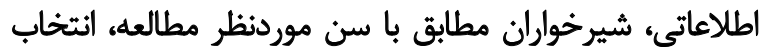

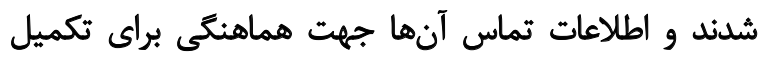

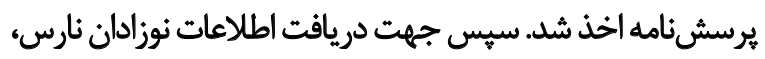

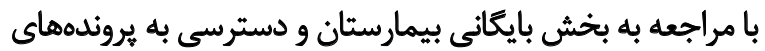
آنها، اطلاعات تماس اين كروه از نوزادان كسب شئد

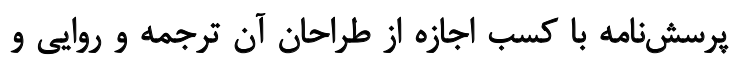

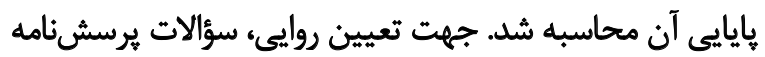

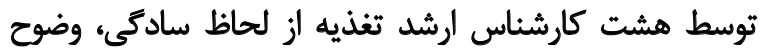

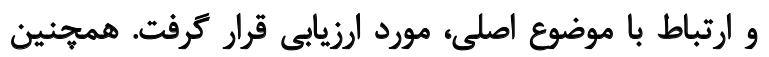

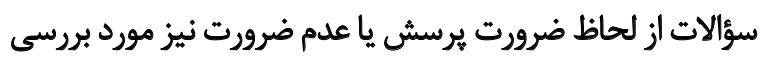

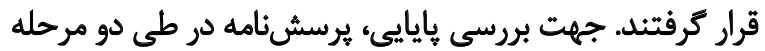

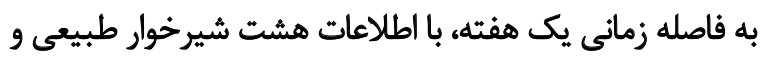

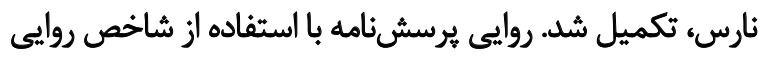

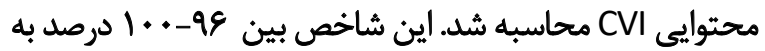

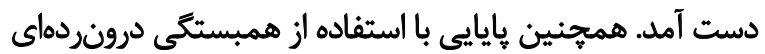

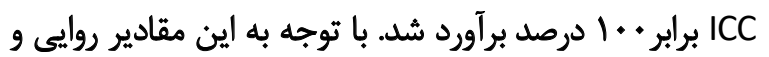

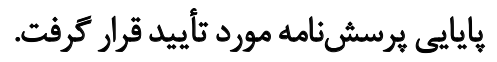




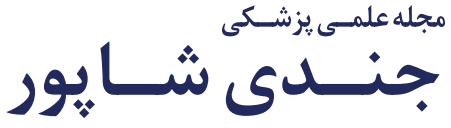

جدول (. مشخصات جمعيتشئاختى آزمودنى ها

\begin{tabular}{|c|c|c|}
\hline \multicolumn{2}{|c|}{ وضعيت } & \multirow{2}{*}{ جنسيث } \\
\hline طييعى & 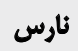 & \\
\hline$n$ & 19 & نهتر \\
\hline re & 川 & يسر \\
\hline$\pi$ & $W / \pi$ & ميانكين مدت زمان بسترى در بخش مراقبتهاي ويزهن نوزادان \\
\hline.$/ \%$ & $\mid r / \Delta V$ & ميانكين سن دستيايى يه تغذيه دهانى مستثل در بدو تولد \\
\hline
\end{tabular}

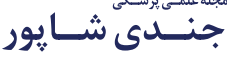

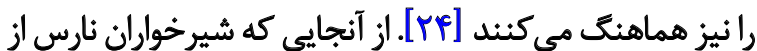

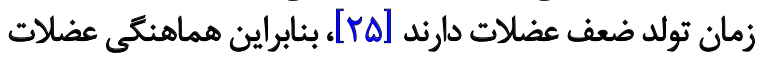

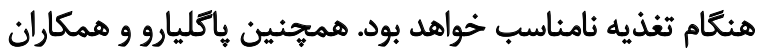

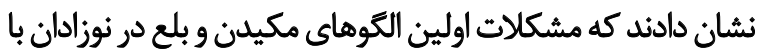

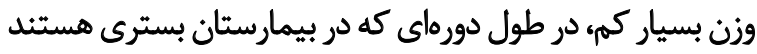

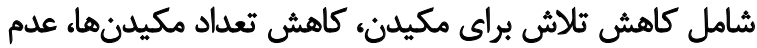

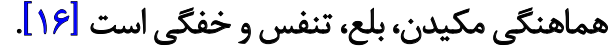

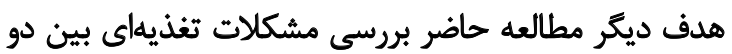

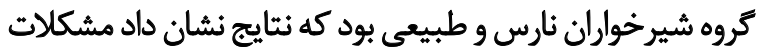

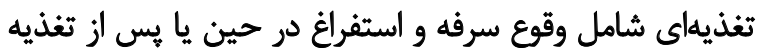

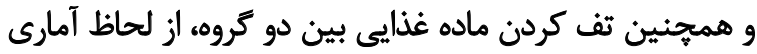

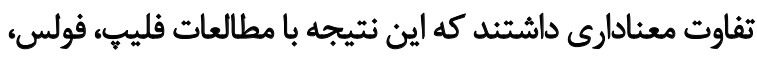

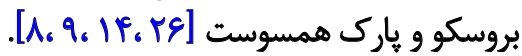
مطالعه فيليب و همكارانش نشان داد كه مشكلات تغذيهاي تا تأنان

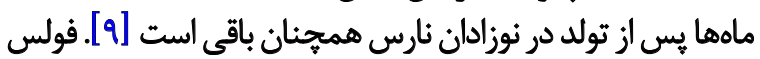

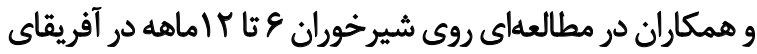

\begin{tabular}{|c|c|c|}
\hline \multicolumn{2}{|c|}{ تعداد (درصد) } & \multirow{2}{*}{ رفتار تغذيه اي } \\
\hline رفتار هر شيرخواران طييعى & رفتار ور شيرخواران نارس & \\
\hline $8 \cdot(1 \cdot)$. & rq(q)/9) & آيا شيرخوار درطى تغذيه سر را به سمت نوك يستان/ بطرى/ قاشق مى خرخاند؟ \\
\hline $8+(1+0)$ & $r+(1+\infty)$ & آيا شيرخوار درطى تغذيه عمل مكيدن را دارد؟ \\
\hline $9 \cdot(1 \cdot 0)$ & $r+(1+\cdots)$ & بيلعدي شيرخوار مىتواند نفسش را نكه دارد و طى يك بلع موفق ب يا ب بار مايع مكيدشده را \\
\hline$\Delta r(M N)$ & $r \Delta(A r / m)$ & آيا شيرخوار مىتواند غذاهاى مخصوص كودكان مانثد يوره را بخورد؟ \\
\hline$\Delta \Delta(9) / \varepsilon)$ & $M(M / M)$ & آيا شيرخوار با اسثفاده از يك قاشق كوجى نوزادى مى تواثد بخورد؟ \\
\hline$\Delta q(Q W T)$ & $M(N \& / 9)$ & آيا شير خوار به طور فعال از زبان خود براى اتثقال عَذا استفاده مي كند؟ \\
\hline $9 \cdot(1 \cdot)$. & $M(v \cdot)$ & آيا شيرخوار در طى تغذيه مىثواند زبانش را روى سخت كام بكشد؟ \\
\hline$\Delta f\left(q_{*}\right)$ & $19(9 \% / \%)$ & آيا شيرخوار قاشق را با لب بالا تميز معكند؟ \\
\hline
\end{tabular}

طبيعى با شيرخواران نارس عماهه اصلاحشده شهر زاهدان بود. در اين مطالعه دو كروه شيرخواران نارس و طبيعى به لحاظ

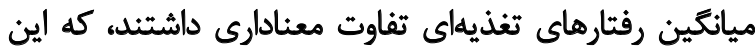

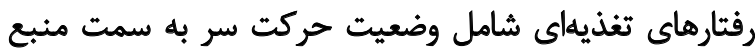

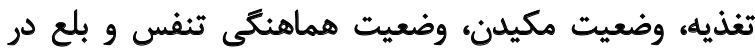

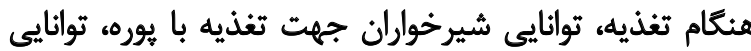

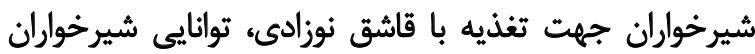

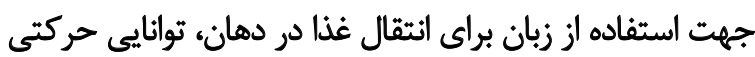

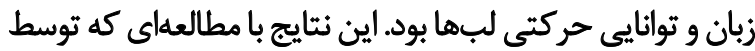

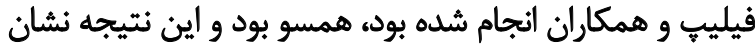

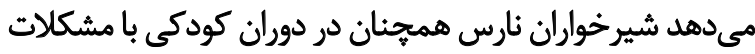

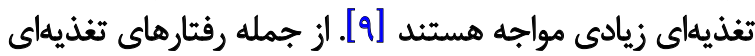

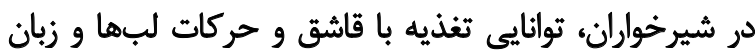
جهت تسهيل تغذيه است كه مطالعه دلنى در اين رابطه نشان

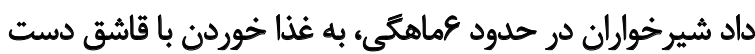

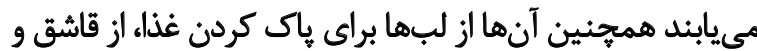

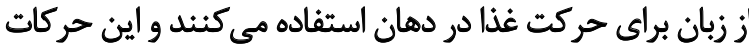


جدول سّ. فراوائى مشكلات تغذيه دو كروه شيرخواران طبيعى و نارس

\begin{tabular}{|c|c|c|c|}
\hline \multirow{2}{*}{$\mathbf{P}$} & \multicolumn{2}{|c|}{ تعداد (درصد) } & \multirow{2}{*}{ مشكلات تغذيهاى } \\
\hline & مشكل در شيرخواران طييعى & مشكل در شيرخواران ثارس & \\
\hline.$\% \circ \mathrm{Fr}$ & IY(YNY) & $11(r e / 9)$ & سرفه در حين خوردن يوره \\
\hline $.1 .9 Y$ & $1 \otimes(T \Delta)$ & $g(r \cdot)$ & استفراغ در حين يا يس از خوردن يوره \\
\hline.$\%$ pr & $Q(N)$ & $11(r e p 9)$ & تف كردن در حين تغذيه \\
\hline
\end{tabular}

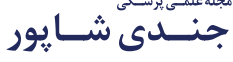

جون اين الكو با الكوى قبلى مكيدن و بلع براى او متفاوت است،

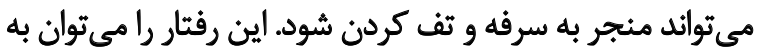

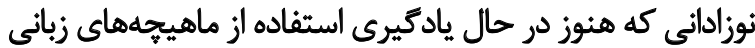

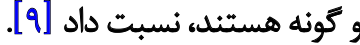

هدف بعدى بررسى نده در اين مطالعه مقايسه بازه زمانى

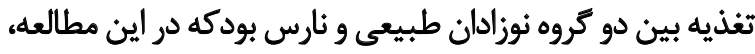

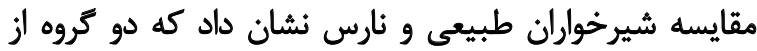

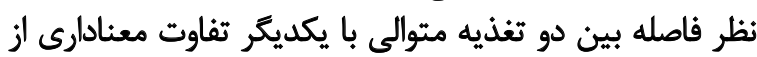

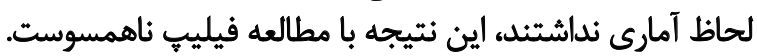

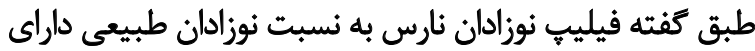

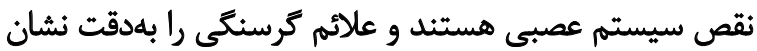

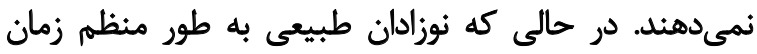

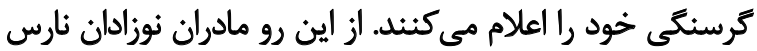

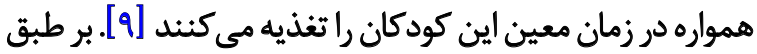

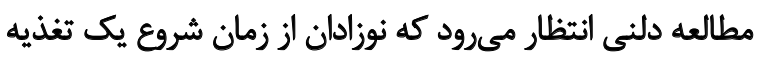

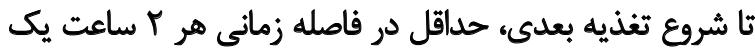

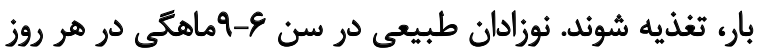

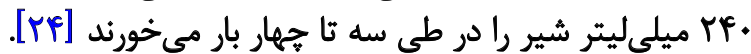

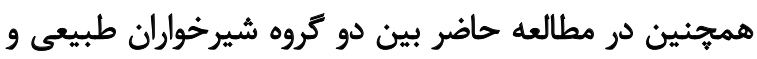

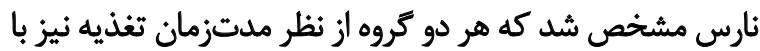

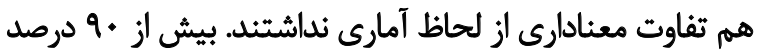

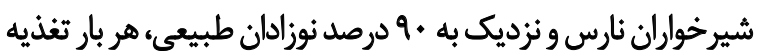

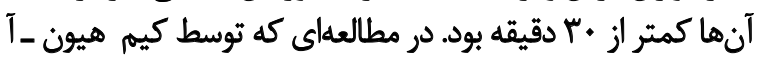

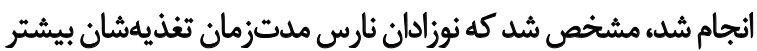

جنوبي دريافتند، /ه/ودرصد آنها مشكلات تغذيهاى شامل امتناع

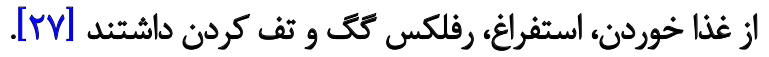

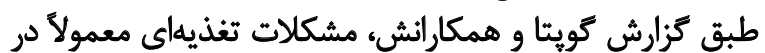

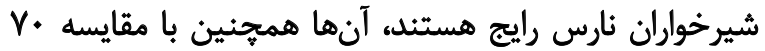

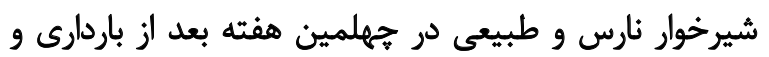

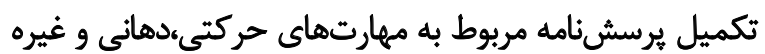

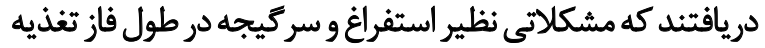

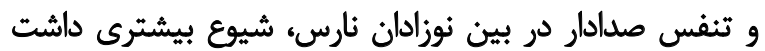

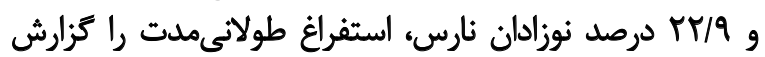

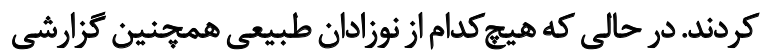

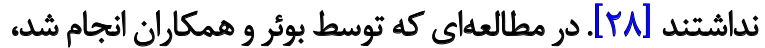

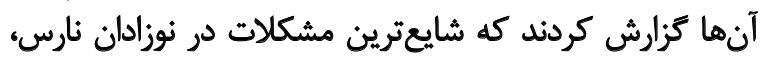

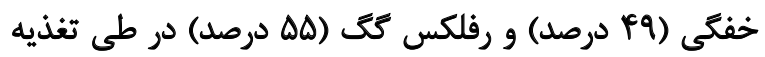

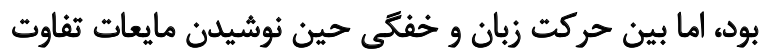

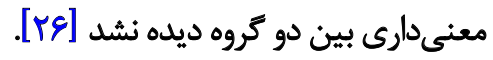
به كزارش جانسون و همكاران، در هنكام معرفى غذاهاى جامد ندامد

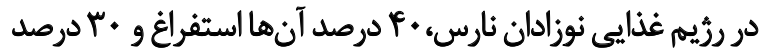

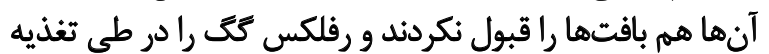

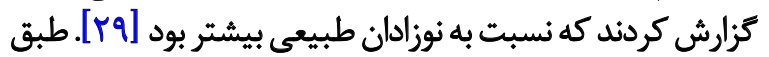

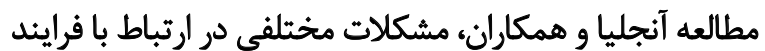

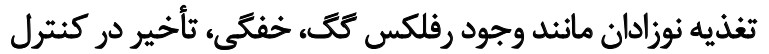

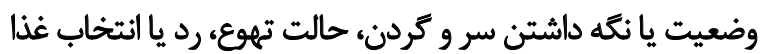

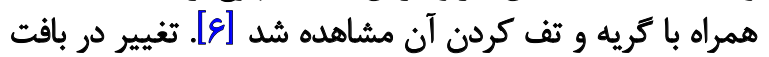

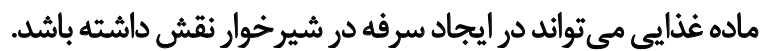

\begin{tabular}{|c|c|c|c|c|}
\hline \multirow{2}{*}{$\mathbf{P}$} & \multicolumn{2}{|c|}{ تعداد } & \multirow{2}{*}{ مدت زمان } & \multirow{2}{*}{ زمان تغذيه } \\
\hline & در شيرخواران طبيعى & در شيرخواران ثارس & & \\
\hline $.10 \%$ & ar & rA & كمتر از •r دقيقه & \multirow{2}{*}{ هدتزمان لازم براي شير بار تغذيه } \\
\hline 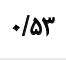 & $\wedge$ & $r$ & بيشتر از هب دقيقه & \\
\hline$\cdot 11$ & pi & $r$ & هر ז-" ساعت & \multirow[b]{2}{*}{ تعداد دفعات تغذيه در شبائهروز } \\
\hline$\cdot / 1$ & 19 & 8 & هر با-4 ساعت & \\
\hline
\end{tabular}




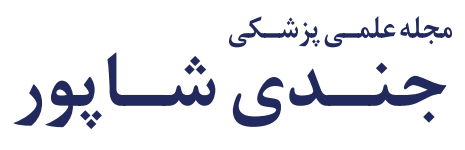

جدول ه. مقايسه وزنَّيرى بين دو كروه شيرخواران ثارس و طبيعى

\begin{tabular}{|c|c|c|c|c|c|}
\hline \multirow{2}{*}{$\mathbf{P}$} & \multicolumn{4}{|c|}{ كيلوكرم } & \multirow{2}{*}{ كروه } \\
\hline & 7 7مكي & عماهكي & זماهكي & بدو تولد & \\
\hline .10 .1 & V/Ta & s/rq & $r / 9$. & $r / . r$ & شيرخواران طبيعى \\
\hline .10 .1 & $\Delta / \circ \Delta$ & r/As & T/ES & VEA & شير خواران نارس \\
\hline
\end{tabular}

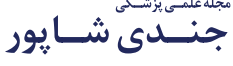

بعدى با حجم نمونه بالاترى انجام شود. با توجه به وقوع بيشتر

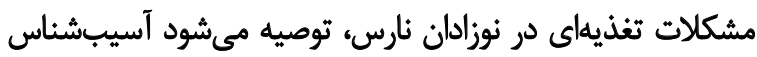

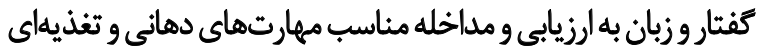

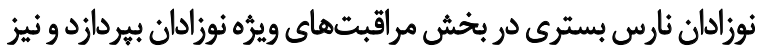

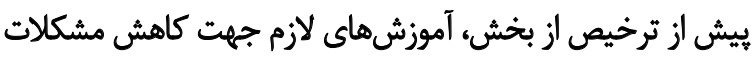

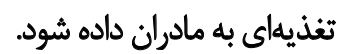

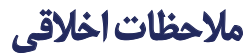 \\ يبيروى از اصول اخلاق بثوهش}

جهت هماهنتى هاى لازم با والدين تماس ترفته شد و يَيش از

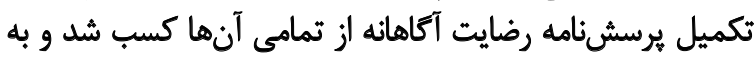

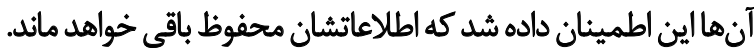

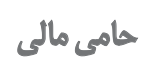

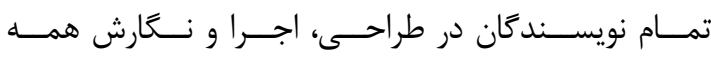

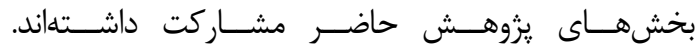

$$
\text { مشاركت نويسندكان }
$$

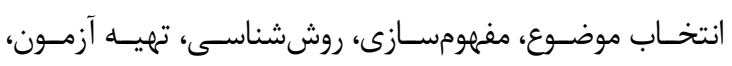

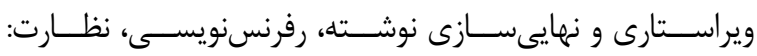

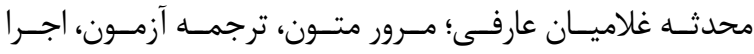

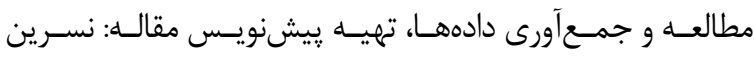

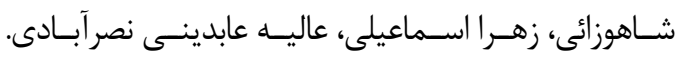

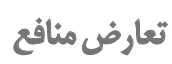

بنابر اظهار نويسندكان اين مقاله تعارض منافع ندارد.

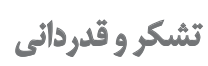

ازئ جناب آقاى دكتر محمد كاظم مؤمنى، رياست محترم

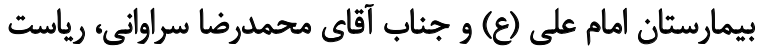

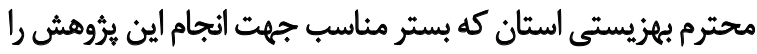

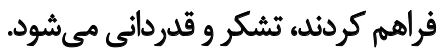

ز زاك ادقيقه بود كه طولانى شدن اين زمان به علت مشكلات حسى

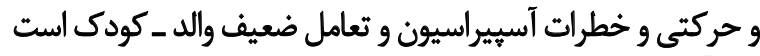

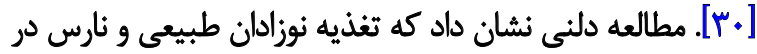

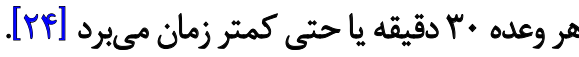

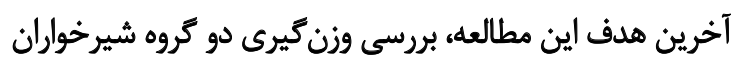

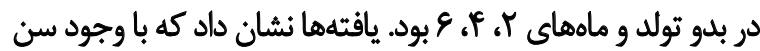

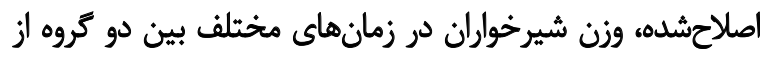

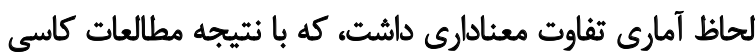

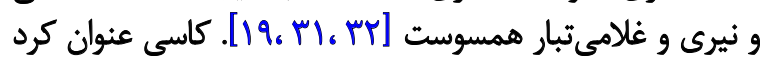

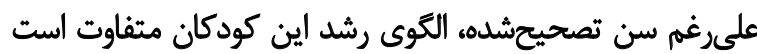

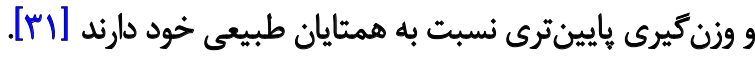

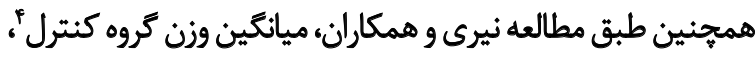

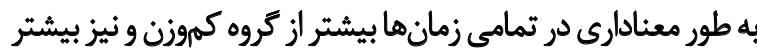

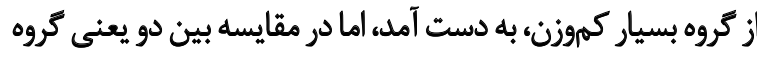

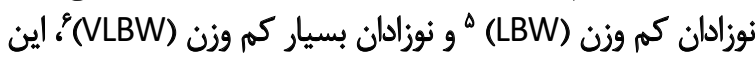

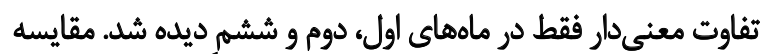

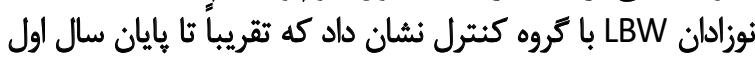

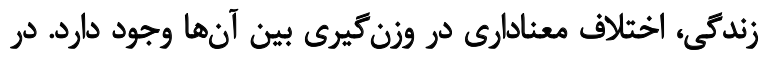

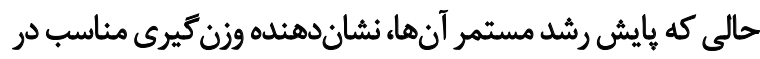

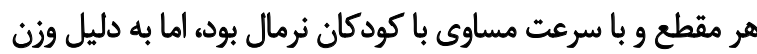

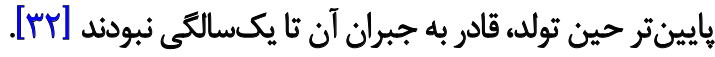

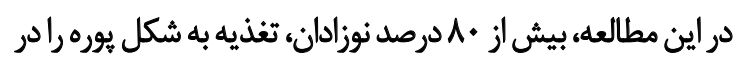

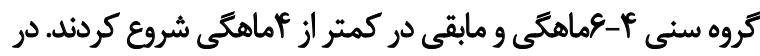

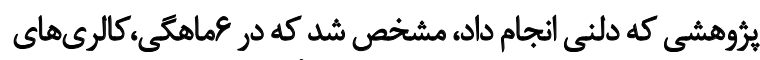

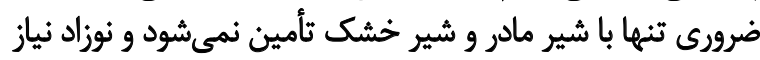

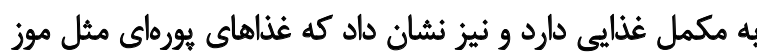

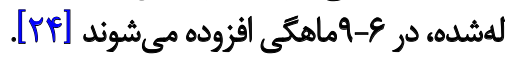

\section{تتيجهئيرى}

نتايج اين مطالعه نشان داد شيرخواران نارس در مقايسه با بإن

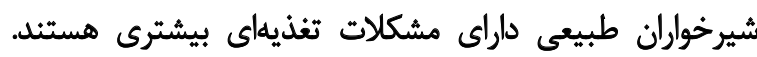

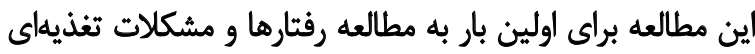

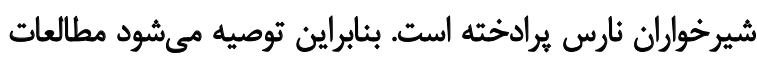

4. Normal Birth Weight (NBW)

5. Low Birth Weight (LBW)

6. Very Low Birth Weight (VLBW) 


\section{Refrence}

[1] Seiiedi-Biarag L, Mirghafourvand M. The effect of massage on feeding intolerance in preterm infants: A systematic review and meta-analysis study. Ital J Pediatr. 2020; 46(1):52. [DOI:10.1186/s13052-020-0818-4] [PMID] [PMCID]

[2] Assadollah-Pour F, Soleymani-Far F, Yadegari F, Younesian Sh. [The effect of nonnutritive sucking on achievement of full oral feeding in preterm infants (Persian)]. Arch Rehabil. 2013; 13:121-7. http://rehabilitationj.uswr.ac.ir/article-1-1090-en. html

[3] Hosseini SS, Baniasadi H, Pouraboli B. [Stressors of parents of hospitalized preterm infants: a study in neonatal intensive care unit of Afzalipour Hospital, Kerman, Iran (Persian)]. J Health Dev. 2015; 4(4):337-48. http://eprints.kmu.ac.ir/27411/

[4] Yonesian Sh, Yadegari F, Soleimani F, Karimlou M. [Effect of Beckman Oral stimulation program on time to attainment of independent oral feeding in preterm infants in NICU (Persian)]. Arch Rehabil. 2011; 11:65-72. http://rehabilitationj.uswr.ac.ir/ article-1-717-en.html

[5] Díaz PF, Valdebenito MR. The transition from tube to nipple in the premature newborn. Newborn Infant Nurs Rev. 2007; 7(2):114-9. [DOI:10.1053/j.nainr.2007.03.003]

[6] Fernández Gallardo MA, Rojas Contreras DP, Vargas Keith JF. [Development of feeding skills in preterm infants: A critical literature review (English-Spanish)]. Rev CEFAC. 2017; 19(4):539-50. [DOI:10.1590/1982-021620171946417]

[7] Hwang YS, Vergara E, Lin CH, Coster WJ, Bigsby R, Tsai WH. Effects of prefeeding oral stimulation on feeding performance of preterm infants. Indian J Pediatr. 2010; 77(8):869-873. [DOI:10.1007/s12098-010-0001-9] [PMID]

[8] Park J, Thoyre SM, Pados BF, Gregas M. Symptoms of feeding problems in preterm-born children at 6 months to 7 years old. $J$ Pediatr Gastroenterol Nutr. 2019; 68(3):416-21. [DOI:10.1097/ MPG.0000000000002229] [PMID]

[9] Philip AK, Vijay Kumar KV. Comparison of feeding behaviours in term infants and preterm infants ( 30 to 34 weeks) at six months corrected age. J Nepal Paediatr Soc. 2016; 35(2):202-5. [DOI:10.3126/jnps.v35i2.12844]

[10] Medoff-Cooper B, McGrath JM, Shults J. Feeding patterns of full-term and preterm infants at forty weeks postconceptional age. J Dev Behav Pediatr. 2002; 23(4):231-6. [DOI:10.1097/00004703-200208000-00007] [PMID]

[11] Arvedson J, Clark H, Lazarus C, Schooling T, Frymark T. Evidence-based systematic review: effects of oral motor interventions on feeding and swallowing in preterm infants. Am J Speech Lang Pathol. 2010; 19(4):321-40. [DOI:10.1044/10580360(2010/09-0067)]

[12] Amaizu N, Shulman R, Schanler R, Lau C. Maturation of oral feeding skills in preterm infants. Acta Paediatr. 2008; 97(1):61-7. [DOI:10.1111/j.1651-2227.2007.00548.x] [PMID] [PMCID]

[13] Lau Ch. Oral feeding in the preterm infant. NeoReviews. 2006; 7(1):e19-27. [DOI:10.1542/neo.7-1-e19]
[14] Brusco TR, Delgado SE. Characterization of the feeding development of preterm infants between three and twelve months. Rev CEFAC. 2014;16(3):917-928. https://www.redalyc.org/ pdf/1693/169331564025_2.pdf

[15] Park J, McComish C, Pados BF, Estrem HH, Thoyre SM. Changes in symptoms of problematic eating over 6 months in infants and young children. Infants Young Child. 2018; 31(4):297-309. [DOI:10.1097/IYC.0000000000000128]

[16] Pagliaro CL, Bühler KEB, Ibidi SM, Limongi SCO. [Dietary transition difficulties in preterm infants: Critical literature review (Portuguese)]. J Pediatr (Versão em Português). 2016; 92(1):714. [DOI:10.1016/j.jpedp.2015.11.001]

[17] Pridham K, Steward D, Thoyre S, Brown R, Brown L. Feeding skill performance in premature infants during the first year. Early Hum Dev. 2007; 83(5):293-305. [DOI:10.1016/j.earlhumdev.2006.06.004] [PMID]

[18] Eivan Bagha R, Hosseini MB, Ghojazadeh M, Amirshahi M. [Continuation rate and reasons of discontinuation of breastfeeding in the preterm infants of mothers admitted Alzahra Hospital of Tabriz (Persian)]. Nurs Midwifery J. 2007; (5):21-8. https://www.magiran.com/paper/738046

[19] Gholamitabar Tabari M, Heidarzadeh M, Sattarzadeh N, Kooshavar H. [Performing and evaluation of breast feeding education program on exclusive breastfeeding and growth indices of preterm infant at 4 months after birth (Persian)]. J Babol Univ Med Sci. 2011; 13(2):57-62. http://jbums.org/ article-1-3742-fa.html

[20] Ghomi H, Yadegari F, Soleimani F, Knoll BL, Noroozi M, Mazouri A. The effects of Premature Infant Oral Motor Intervention (PIOMI) on oral feeding of preterm infants: A randomized clinical trial. Int J Pediatr Otorhinolaryngol. 2019; 120:202-9. [DOI:10.1016/j.ijporl.2019.02.005] [PMID]

[21] Asadollahpour F, Yadegari F, Soleimani F, Younesian Sh. [The effect of Beckman prefeeding oral stimulation program on feeding performance of preterm infants (Persian)]. J Res Rehabil Sci. 2013; 9(4):683-92. http://jrrs.mui.ac.ir/index.php/jrrs/ article/view/952

[22] Soleimani F, Azari N, Kraskian-Mojembari A, Vameghi R, Shahshahani-Pour S, Sajedi F. [Developing of the Persian version of Bayley scales of infant and toddlers development screening test and determine its validity and reliability (Persian)]. Arch Rehabil. 2014; 14(S2):18-29. http://rehabilitationj.uswr.ac.ir/ article-1-1418-en.html

[23] American Speech-Language-Hearing Association. Roles of speech-language pathologists in the neonatal intensive care unit: Technical report [Internet]. 2004 [Updated 2003 October]. Available from: https://docuri.com/download/10-y-11uci_59ae494af581710a620168d9_pdf

[24] Delaney AL, Arvedson JC. Development of swallowing and feeding: Prenatal through first year of life. Dev Disabil Res Rev. 2008; 14(2):105-17. [DOI:10.1002/ddrr.16] [PMID]

[25] Skaaning D, Kronborg H, Brødsgaard A, Solmer R, Pryds O, Carlsen EM. No long-term effect of oral stimulation on the intra-oral vacuum in healthy premature infants. Acta Paediatrica. 2020; 109(10):2025-32. [DOI:10.1111/apa.15289] 
[26] den Boer SL, Schipper JA. Feeding and drinking skills in preterm and low birth weight infants compared to full term infants at a corrected age of nine months. Early Hum Dev. 2013; 89(6):445-7. [DOI:10.1016/j.earlhumdev.2012.12.004] [PMID]

[27] Fuls N, Krüger E, van der Linde J. Feeding characteristics of infants in a lower-middle-income country. J Paediatr Child Health. 2020; 56(7):1083-9. [DOI:10.1111/jpc.14823] [PMID]

[28] Gupta G, Vijay Kumar KV, Anitha R, Ninan B. Feeding performance in preterm infants and full term infants at 40 weeks postmenstrual age. J Nepal Paediatr Soc. 2016; 36(2):136-40. [DOI:10.3126/jnps.v36i2.14621]

[29] Johnson S, Matthews R, Draper ES, Field DJ, Manktelow BN, Marlow N, et al. Eating difficulties in children born late and moderately preterm at $2 \mathrm{y}$ of age: A prospective populationbased cohort study-3. Am J Clin Nutr. 2016; 103(2):406-14. [DOI:10.3945/ajcn.115.121061]

[30] Kim HA, Kim MJ. Perinatal outcomes of preterm twins< 34 weeks after IVF pregnancies versus natural conception: Same oral feeding tolerance? J Korean Soc Neonatol. 2012;19(2):84-90. [DOI:10.5385/jksn.2012.19.2.84]

[31] Casey PH, Kraemer HC, Bernbaum J, Yogman MW, Sells JC. Growth status and growth rates of a varied sample of low birth weight, preterm infants: A longitudinal cohort from birth to three years of age. J Pediatr. 1991; 119(4):599-605. [DOI:10.1016/S0022-3476(05)82414-X]

[32] Nayeri FS, Kheradpisheh N, Shariat M, Akbari Asbagh P. [A comparison between the growth trend of normal and low birth weight newborns during the first year of life (Persian)]. Tehran Univ Med J. 2009; 67(4):296-302. http://tumj.tums. ac.ir/article-1-464-en.html 
This Page Intentionally Left Blank 